\title{
Weak Solutions and Optimal Control for Multivalued Stochastic Differential Equations
}

\author{
Adrian Zălinescu
}

\begin{abstract}
In this paper we first prove the existence of a weak solution to a finite dimensional multivalued stochastic differential equation of the form

$$
d X_{t}+A\left(X_{t}\right) d t \ni b(t, X) d t+\sigma(t, X) d B_{t}, \quad t \in[0, T],
$$

where $A$ is a maximal monotone operator, and the coefficients $b$ and $\sigma$ are continuous functionals of the state variable. The main tool used is the martingale problem approach.

Secondly we are concerned with a control problem where the system is driven by a similar equation and the control policy takes its values in a compact space. Using the martingale problem formulation, we show the existence of an optimal relaxed control. Under some supplementary hypotheses of convexity on the coefficients, we prove the existence of an optimal control for the initial problem.
\end{abstract}

Mathematics Subject Classification (2000). Primary 60H99; Secondary 93E20. Keywords. Multivalued stochastic differential equations, weak solutions, optimal control.

\section{Introduction}

The first aspect we treat in this paper is the problem of the existence of a solution to the following multivalued stochastic differential equation (MSDE), driven by a standard, $d^{\prime}$-dimensional Brownian motion $B$ :

$$
d X_{t}+A\left(X_{t}\right) d t \ni b(t, X) d t+\sigma(t, X) d B_{t}, \quad t \in[0, T],
$$

where $A$ is a maximal monotone operator on $\mathbb{R}^{d}$ and the functions $b, \sigma$ are defined on $[0, T] \times C\left([0, T] ; \mathbb{R}^{d}\right)$ with values in $\mathbb{R}^{d}$, respectively $\mathbb{R}^{d \times d^{\prime}}$.

Existence and uniqueness of strong solutions to such equations for Lipschitzcontinuous coefficients $b$ and $\sigma$ were proved by several authors. We first mention [8],

Work supported by Romanian Academy under contract GAR 18/2007. 
in which the problem was considered in finite dimensional spaces. The infinite dimensional case was investigated in [4] for the particular situation where $A$ was the subdifferential of a convex, proper, lower semicontinuous function. Using the method of singular inputs, this constraint was removed in [13].

We are interested in solving equation (1.1) for only continuous dependence in the state variable of the drift, respectively diffusion coefficients $b$ and $\sigma$. But even in the case $A \equiv 0$, it might not exist a strong solution. The most usual way to deal with this problem is to conceive solutions in the weak sense. A variation on this approach, developed in [14], formulates the search for the law of a diffusion process with given drift and diffusion coefficients in terms of a martingale problem. We adapt to our case this martingale formulation, which has the advantage of being well suited for the continuity and weak convergence arguments which yield the existence result (Theorem 3.6). This will be the subject of the third section.

In the last section we are concerned with an optimal control problem, governed by the state equation

$$
d X_{t}+A\left(X_{t}\right) d t \ni b\left(t, X, u_{t}\right) d t+\sigma\left(t, X, u_{t}\right) d B_{t}, \quad t \in[0, T],
$$

where this time $b$ and $\sigma$ are allowed to depend on the stochastic control policy $u$, which takes values in a compact metric space. Here, a control is to be understood in a weak sense. We must emphasize that the main difference between weak and strong controls consists in the fact that the latter are constructed on a a priori given space with its filtration.

Our purpose is to find a control, the so-called optimal control, which minimizes the cost functional

$$
\mathbb{E}\left[\int_{0}^{T} g\left(t, X, u_{t}\right) d t+h\left(X_{T}\right)\right],
$$

the functions $g$ and $h$ being known in the literature as the running cost, respectively the terminal cost.

Adapting the ideas of [10] to our framework, we prove the existence of an optimal control for an extended problem by allowing the controls to take values in a larger space than the initial one. Under a supplementary convexity assumption on $b, \sigma$ and $g$, we are able to go backward and solve the original problem.

\section{Preliminaries}

\subsection{Maximal monotone operators}

Let us recall the definition and some properties of maximal monotone operators. The interested reader can find a general development on this topic in [6], for example.

A multivalued operator on $\mathbb{R}^{d}$ is a map $A: \mathbb{R}^{d} \rightarrow 2^{\mathbb{R}^{d}}$, with $2^{\mathbb{R}^{d}}$ denoting the class of all subsets of $\mathbb{R}^{d}$. Such an operator can be identified with its graph:

$$
\operatorname{Gr} A:=\left\{\left(x, x^{*}\right) \in \mathbb{R}^{d} \times \mathbb{R}^{d} \mid x^{*} \in A(x)\right\} .
$$


We also define the domain of $A$ :

$$
\operatorname{Dom} A:=\left\{x \in \mathbb{R}^{d} \mid A(x) \neq \emptyset\right\} .
$$

A monotone operator on $\mathbb{R}^{d}$ is a multivalued operator $A$ such that

$$
\left\langle x-y, x^{*}-y^{*}\right\rangle \geq 0, \quad \forall\left(x, x^{*}\right), \quad\left(y, y^{*}\right) \in \operatorname{Gr} A,
$$

where $\langle\cdot, \cdot\rangle$ is the usual scalar product in $\mathbb{R}^{d}$.

The class of monotone operators on $\mathbb{R}^{d}$ can be ordered by the inclusion between their graphs:

$$
A \preceq \tilde{A} \Leftrightarrow A(x) \subseteq \tilde{A}(x), \quad \forall x \in \mathbb{R}^{d} \Leftrightarrow \operatorname{Gr} A \subseteq \operatorname{Gr} \tilde{A} .
$$

A maximal element with respect to this order is called a maximal monotone operator on $\mathbb{R}^{d}$. According to this definition, a monotone operator $A$ is maximal monotone if and only if, for every $\left(x, x^{*}\right) \in \mathbb{R}^{d} \times \mathbb{R}^{d}$,

$$
\left(x, x^{*}\right) \in \operatorname{Gr} A \Leftrightarrow\left\langle x-y, x^{*}-y^{*}\right\rangle \geq 0, \quad \forall\left(y, y^{*}\right) \in \operatorname{Gr} A .
$$

If $A$ is a maximal monotone operator on $\mathbb{R}^{d}$, then $\operatorname{Int}(\operatorname{Dom} A), \overline{\operatorname{Dom} A}{ }^{\dagger}$ are convex sets and

$$
\operatorname{Int}(\operatorname{Dom} A)=\operatorname{Int}(\overline{\operatorname{Dom} A}) .
$$

Moreover, for every $x \in \mathbb{R}^{d}, A(x)$ is a closed convex set of $\mathbb{R}^{d}$.

An essential property of a maximal monotone operator $A$ is the local boundedness of $A$ on $\operatorname{Int}(\operatorname{Dom} A)$, i.e. $\bigcup_{x \in \mathbb{K}} A(x)$ is bounded for every compact set $\mathbb{K} \subseteq \operatorname{Int}(\operatorname{Dom} A)$.

A most remarkable example of maximal monotone operator is the subdifferential of a proper, convex, lower semicontinuous (l.s.c.) function $\left.\left.\varphi: \mathbb{R}^{d} \rightarrow\right]-\infty,+\infty\right]$, defined by

$$
\partial \varphi(x):=\left\{x^{*} \in \mathbb{R}^{d} \mid\left\langle y-x, x^{*}\right\rangle+\varphi(x) \leq \varphi(y), \forall y \in \mathbb{R}^{d}\right\} .
$$

If $d=1$, every maximal monotone operator on $\mathbb{R}^{d}$ can be expressed in this way.

As a particular case, we consider the indicator function of a closed convex set $\mathbb{K} \subseteq \mathbb{R}^{d}$,

$$
I_{\mathbb{K}}(x):= \begin{cases}0, & x \in \mathbb{K} \\ +\infty, & x \in \mathbb{R}^{d} \backslash \mathbb{K} .\end{cases}
$$

Then the subdifferential of this proper, convex, l.s.c. function is

$$
\partial I_{\mathbb{K}}(x)= \begin{cases}\{0\}, & x \in \operatorname{Int} \mathbb{K} ; \\ \prod_{x}, & x \in \operatorname{Fr} \mathbb{K} ; \\ \emptyset, & x \in \mathbb{R}^{d} \backslash \mathbb{K},\end{cases}
$$

where $\Pi_{x}$ denotes the exterior normal cone to $\mathbb{K}$ in $x$.

\footnotetext{
${ }^{\dagger}$ Given a subset $S$ of a topological space, we denote by $\operatorname{Int} S, \bar{S}$, and $\operatorname{Fr} S$, the interior, the closure,
} respectively the boundary of $S$. 


\subsection{Strong solutions of MSDEs}

Throughout the paper we consider some fixed $T>0, d, d^{\prime} \in \mathbb{N}^{*}$ and a maximal monotone operator $A$ on $\mathbb{R}^{d}$ which satisfies

$$
\operatorname{Int}(\operatorname{Dom} A) \neq \emptyset .
$$

For the sake of simplicity, let us denote $\mathbf{W}:=C\left([0, T] ; \mathbb{R}^{d}\right)$. On $\mathbf{W}$ we set the $\sigma$-algebra $\mathcal{B}_{t}:=\Psi_{t}^{-1}(\mathcal{B}(\mathbf{W}))$, where $t \geq 0$ and $\Psi_{t}: \mathbf{W} \rightarrow \mathbf{W}$ is defined by $\Psi_{t}(x):=x(t \wedge \cdot)$. We call $\left\{\mathcal{B}_{t}\right\}_{t>0}$ the canonical filtration on $\mathbf{W}$. We also denote by $|\cdot|$ and $\|\cdot\|_{\mathbf{W}}$ the Euclidian norm in $\mathbb{R}^{d}$, respectively the supremum norm in $\mathbf{W}$.

For every function $\eta:[0, T] \rightarrow \mathbb{R}^{d}$, we set $\|\eta\|_{\mathbf{B V}}:=\left(\sum_{i=1}^{d}\left(\operatorname{var} \eta_{i}\right)^{2}\right)^{1 / 2}$, where $\operatorname{var} \eta_{i}$ is the total variation on $[0, T]$ of the $i^{\text {th }}$ component of $\eta$. The space of $d$-dimensional functions $\eta$ with bounded variation on $[0, T]\left(\right.$ i.e. $\left.\|\eta\|_{\mathbf{B V}}<+\infty\right)$ is denoted $B V\left([0, T] ; \mathbb{R}^{d}\right)$.

Let $\left(\Omega, \mathcal{F}, P,\left\{\mathcal{F}_{t}\right\}_{t \geq 0}\right)$ be a stochastic basis $(i . e .(\Omega, \mathcal{F}, P)$ is a complete probability space and the filtration $\left\{\mathcal{F}_{t}\right\}_{t \geq 0}$ satisfies the usual conditions $), B$ a standard, $d^{\prime}$-dimensional Brownian motion with respect to this basis and $b:[0, T] \times \Omega \times \mathbf{W} \rightarrow$ $\mathbb{R}^{d}, \sigma:[0, T] \times \Omega \times \mathbf{W} \rightarrow \mathbb{R}^{d \times d^{\prime}}$ two nonanticipative processes, i.e. progressively measurable processes with respect to the filtration $\left\{\mathcal{F}_{t} \otimes \mathcal{B}_{t}\right\}_{t \geq 0}$.

We say that a pair of $d$-dimensional stochastic processes $\left(X_{t}, K_{t}\right)_{t \in[0, T]}$ is a (strong) solution of equation (1.1) if

$$
\left\{\begin{aligned}
\text { i) } & X \text { and } K \text { are continuous and }\left\{\mathcal{F}_{t}\right\}_{t \geq 0^{-a d a p t e d}} \\
\text { ii) } & K \text { has bounded variation and } K_{0}=0, \quad \text { a.s. } \\
\text { iii) } & \int_{0}^{T}\left(|b(t, X)|+|\sigma(t, X)|^{2}\right) d t<+\infty, \quad \text { a.s. } \\
\text { iv) } & X_{t}+K_{t}=X_{0}+\int_{0}^{t} b(s, X) d s+\int_{0}^{t} \sigma(s, X) d B_{s}, \quad \forall t \in[0, T], \quad \text { a.s. } \\
\text { v) } & \int_{s}^{t}\left\langle X_{r}-z, d K_{r}-z^{*} d r\right\rangle \geq 0,0 \leq s \leq t \leq T, \quad\left(z, z^{*}\right) \in \operatorname{Gr} A, \quad \text { a.s. }
\end{aligned}\right.
$$

The last condition formally means that " $d K_{t} \in A\left(X_{t}\right) d t$ " a.s. In fact, for $x \in \mathbf{W}$, let $\mathcal{A}(x)$ be defined as the set of functions $\eta \in \mathbf{W} \cap B V\left([0, T] ; \mathbb{R}^{d}\right)$ which satisfy

$$
\int_{s}^{t}\left\langle x(r)-z, d \eta(r)-z^{*} d r\right\rangle \geq 0, \quad \forall 0 \leq s \leq t \leq T, \quad \forall\left(z, z^{*}\right) \in \operatorname{Gr} A .
$$

Then we have the following result.

Proposition 2.1. Let $x \in \mathbf{W}$ and $\eta \in \mathbf{W} \cap B V\left([0, T] ; \mathbb{R}^{d}\right)$. The following statements are equivalent:

i) $\eta \in \mathcal{A}(x)$;

ii) for all $0 \leq s \leq t \leq T$, and $\left(y, y^{*}\right) \in \mathbf{W} \times \mathbf{W}$ such that $\left(y(r), y^{*}(r)\right) \in \operatorname{Gr} A$, $\forall r \in[0, T]$, it holds

$$
\int_{s}^{t}\left\langle x(r)-y(r), d \eta(r)-y^{*}(r) d r\right\rangle \geq 0
$$


iii) for all $0 \leq s \leq t \leq T, \tilde{x} \in \mathbf{W}$, and $\tilde{\eta} \in \mathcal{A}(\tilde{x})$ :

$$
\int_{s}^{t}\langle x(r)-\tilde{x}(r), d \eta(r)-d \tilde{\eta}(r)\rangle \geq 0 .
$$

Implication "ii) $\Rightarrow \mathrm{i}$ )" is obvious, while "i) $\Rightarrow \mathrm{ii}$ )" follows from the approximation of continuous functions by step functions (see also Lemma 3.3). Then "ii $\Rightarrow$ iii)" is just a reformulation of $[8$, Lemma 4.1]. Finally, assertion "iii) $\Rightarrow$ i)" is an easy consequence of the fact that, with $\left(z, z^{*}\right) \in \operatorname{Gr} A$ and $x(r):=z, \eta(r):=r z^{*}$, $r \in[0, T], \eta$ is an element of $\mathcal{A}(x)$.

Remark. Using the fact that a monotone function is almost everywhere derivable, one can show that $\eta \in \mathcal{A}(x)$ implies that $x(t) \in \operatorname{Dom} A$ for almost all $t \in[0, T]$. Since the definition of strong solutions given in [8] was formulated in terms of ii) and also required that $X_{t} \in \overline{\operatorname{Dom} A}$ a.s., the above result ensures that our definition is equivalent to that one.

Particular case. If $A$ is the subdifferential of a proper, convex, l.s.c. function $\varphi$ : $\left.\left.\mathbb{R}^{d} \rightarrow\right]-\infty,+\infty\right]$, then $\mathcal{A}(x)$ is the set of functions $\eta \in \mathbf{W} \cap B V\left([0, T] ; \mathbb{R}^{d}\right)$ which satisfy

$$
\int_{s}^{t}\langle z-x(r), d \eta(r)\rangle+\int_{s}^{t} \varphi(x(r)) d r \leq(t-s) \varphi(z),
$$

for all $0 \leq s \leq t \leq T$ and $z \in \mathbb{R}^{d}$.

Moreover, if $A=\partial I_{\mathbb{K}}$, where $\mathbb{K} \subseteq \mathbb{R}^{d}$ is a nonempty closed convex set, our problem becomes that of a (normal) reflected diffusion problem (with drift $b$ and diffusion $\sigma$ ) in $\mathbb{K}$.

We will give now some a priori estimations for the solutions of (1.1). The following hypothesis will be needed $(\alpha>0)$ :

$$
|b(t, \omega, x)|+|\sigma(t, \omega, x)| \leq \alpha\left(1+\|x\|_{\mathbf{W}}\right), \quad \forall(t, \omega, x) \in[0, T] \times \Omega \times \mathbf{W} .
$$

Proposition 2.2. Suppose that (2.3) holds. If $(X, K)$ is a solution of equation (1.1) and $p \geq 1$ then we have

$$
\mathbb{E}\left(\|X\|_{\mathbf{W}}+\|K\|_{\mathbf{W}}\right)^{2 p}+\mathbb{E}\|K\|_{\mathbf{B V}}^{p} \leq C\left(1+\mathbb{E}\left|X_{0}\right|^{2 p}\right),
$$

where $C$ is a constant depending only on $d, T, \alpha, p$ and $A$.

We will just sketch the proof because its similitude with the case $A \equiv 0$ (see for example, [12, Problem 5.3.15]).

Proof. Throughout the proof we will use the same letter $C$ for constants depending only on $d, T, \alpha, p$, and $A$.

Let $(X, K)$ be a solution of (1.1), $p \geq 1$, and assume that $\mathbb{E}\left|X_{0}\right|^{2 p}<+\infty$.

We suppose first that $0 \in \operatorname{Int}(\operatorname{Dom} A)$ and $0 \in A(0)$. 
By Itô formula, we have

$$
\begin{aligned}
\left|X_{t}\right|^{2}+2 \int_{0}^{t}\left\langle X_{s}, d K_{s}\right\rangle= & \left|X_{0}\right|^{2}+2 \int_{0}^{t}\left\langle X_{s}, b(s, X)\right\rangle d s \\
& +\int_{0}^{t}|\sigma(s, X)|^{2} d s+2 \int_{0}^{t}\left\langle X_{s}, \sigma(s, X) d B_{s}\right\rangle .
\end{aligned}
$$

By (2.1)-v) and the assumption made on $A$,

$$
\int_{0}^{t}\left\langle X_{r}, d K_{r}\right\rangle \geq 0, \quad \forall t \in[0, T], \quad \text { a.s } .
$$

Therefore, using localization, the inequality of Burkholder-Davis-Gundy, and the inequality of Gronwall, one obtains, just like in the case $A \equiv 0$, that

$$
\mathbb{E}\|X\|_{\mathbf{W}}^{2 p} \leq C\left(1+\mathbb{E}\left|X_{0}\right|^{2 p}\right)
$$

From (2.4) we also obtain

$$
\mathbb{E}\left(\int_{0}^{T}\left\langle X_{s}, d K_{s}\right\rangle\right)^{p} \leq C\left(1+\mathbb{E}\left|X_{0}\right|^{2 p}\right) .
$$

On the other hand, from (2.1)- $v$ ) it follows that a.s., for all $0 \leq s \leq t \leq T$ and $\left(z, z^{*}\right) \in \operatorname{Gr} A$,

$$
\left\langle z, K_{t}-K_{s}\right\rangle \leq \int_{s}^{t}\left\langle X_{r}, d K_{r}\right\rangle+(t-s)\left(|z|+\|X\|_{\mathbf{W}}\right)\left|z^{*}\right| .
$$

The assumption $0 \in \operatorname{Int}(\operatorname{Dom} A)$ implies that there exists $\delta>0$ such that $\overline{B(0 ; \delta)}{ }^{\dagger}$ $\subseteq \operatorname{Int}(\operatorname{Dom} A)$. Since $A$ is locally bounded on $\operatorname{Int}(\operatorname{Dom} A)$ (see $[6]$ ), we have

$$
a_{0}:=\sup \left\{x^{*} \mid \exists x \in \overline{B(0 ; \delta)}: x^{*} \in A(x)\right\}<+\infty .
$$

Therefore, a.s., for all $0 \leq s \leq t \leq T$,

$$
\delta\left|K_{t}-K_{s}\right| \leq \int_{s}^{t}\left\langle X_{r}, d K_{r}\right\rangle+(t-s)\left(\delta+\|X\|_{\mathbf{W}}\right) a_{0}
$$

which yields

$$
\delta\|K\|_{\mathbf{B V}} \leq \int_{0}^{T}\left\langle X_{r}, d K_{r}\right\rangle+T\left(\delta+\|X\|_{\mathbf{W}}\right) a_{0}, \quad \text { a.s } .
$$

According to (2.6), we obtain

$$
\mathbb{E}\|K\|_{\mathbf{B V}}^{p} \leq C\left(1+\mathbb{E}\left|X_{0}\right|^{2 p}\right) .
$$

Let now suppose that $A$ does not satisfy any longer the hypotheses stated at the beginning of the proof. But this case can be reduced to the particular one as follows:

\footnotetext{
${ }^{\dagger}$ As usual, we denote by $B(x ; r)$ the set $\left\{y \in \mathbb{R}^{d}|| x-y \mid<r\right\}$.
} 
Let $x_{0}, x_{0}^{*} \in \mathbb{R}^{d}$ be such that $x_{0} \in \operatorname{Int}(\operatorname{Dom} A)$ and $x_{0}^{*} \in A\left(x_{0}\right)$. We define

$$
\begin{aligned}
\tilde{A}(x) & :=A\left(x+x_{0}\right)-x_{0}^{*}, \quad x \in \mathbb{R}^{d} ; \\
\tilde{b}(t, \omega, x) & :=b\left(t, \omega, x+x_{0}\right)-x_{0}^{*}, \\
\tilde{\sigma}(t, \omega, x) & :=\sigma\left(t, \omega, x+x_{0}\right), \quad(t, \omega, x) \in[0, T] \times \Omega \times \mathbf{W} ; \\
\tilde{X}_{t} & :=X_{t}-x_{0}, \\
\tilde{K}_{t} & :=K_{t}-t x_{0}^{*}, \quad t \in[0, T] .
\end{aligned}
$$

Then $\tilde{A}$ is a maximal monotone operator, $0 \in \operatorname{Int}(\operatorname{Dom} \tilde{A}), 0 \in \tilde{A}(0)$ and $\tilde{b}, \tilde{\sigma}$ satisfy the assumptions of the proposition. It is also easily seen that $(\tilde{X}, \tilde{K})$ is a solution of

$$
d \tilde{X}_{t}+\tilde{A}\left(\tilde{X}_{t}\right) d t \ni \tilde{b}(t, \tilde{X}) d t+\tilde{\sigma}(t, \tilde{X}) d B_{t}, \quad t \in[0, T] .
$$

Then it is just a matter of simple calculations to see that the estimations on $X$ and $K$ also hold.

We state now without proof the main result of existence and uniqueness for strong solutions, which is a slight generalization of [8, Theorem 3]. Suppose that $b$ and $\sigma$ satisfy, for a constant $L>0$ :

$$
\left\{\begin{array}{rlrl}
\text { i) } & |b(t, \omega, x)-b(t, \omega, y)| \leq L\|x-y\|_{\mathbf{W}}, \quad \forall t \in[0, T], \quad \forall x, y \in \mathbf{W}, \quad \text { a.s. } \\
\text { ii) } & |\sigma(t, \omega, x)-\sigma(t, \omega, y)| \leq L\|x-y\|_{\mathbf{W}}, \quad \forall t \in[0, T], \quad \forall x, y \in \mathbf{W}, \quad \text { a.s. } \\
\text { iii) } & \mathbb{E} \int_{0}^{T}\left(|b(t, 0)|^{2}+|\sigma(t, 0)|^{2}\right)<+\infty . &
\end{array}\right.
$$

Theorem 2.3. Let $\xi \in L^{2}\left(\Omega, \mathcal{F}_{0}, P ; \mathbb{R}^{d}\right)$ such that $\xi \in \overline{\operatorname{Dom} A}$, a.s. Under the above assumptions, equation (1.1) has a unique solution with $\xi$ as initial state.

In fact, in [8], the coefficients depended only on the present state of the dynamics, but the proof works as well when we consider the dependence on time and on the past (see also the Remark below the statement of [8, Theorem 3]).

\section{Weak solutions}

Throughout this section, $b:[0, T] \times \mathbf{W} \rightarrow \mathbb{R}^{d}$ and $\sigma:[0, T] \times \mathbf{W} \rightarrow \mathbb{R}^{d \times d^{\prime}}$ are two progressively measurable processes (with respect to the filtration $\left\{\mathcal{B}_{t}\right\}_{t>0}$ ). If $\left(\Omega, \mathcal{F}, P,\left\{\mathcal{F}_{t}\right\}_{t \geq 0}\right)$ is a stochastic basis, $B$ is a standard, $d^{\prime}$-dimensional Brownian motion with respect to this basis and $(2.1)$ holds, we say that $\left(\Omega, \mathcal{F}, P,\left\{\mathcal{F}_{t}\right\}_{t>0}, B\right.$, $X, K)$ is a weak solution of equation (1.1). The probability measure $\mu:=P \circ X_{0}^{-1}$ is called the initial distribution of the solution.

As usual, we impose a growth condition on the coefficients $(\alpha>0)$ :

$$
|b(t, x)|+|\sigma(t, x)| \leq \alpha\left(1+\|x\|_{\mathbf{W}}\right), \quad \forall(t, x) \in[0, T] \times \mathbf{W} .
$$

We shall now show that any class of solutions of equation (1.1) is tight. In order to do this, we will use the following characterization of tightness (cf. [15]): 
Proposition 3.1. A class of probabilities $\mathcal{P}$ on $C\left([0, T] ; \mathbb{R}^{d}\right)$ is tight if and only if

$$
\lim _{a \nearrow \infty} \sup _{P \in \mathcal{P}} P[|x(0)| \geq a]=0
$$

and, for all $\rho>0$,

$$
\lim _{\delta \searrow 0} \sup _{P \in \mathcal{P}} P\left[\sup _{0 \leq t-s \leq \delta}|x(t)-x(s)| \geq \rho\right]=0 .
$$

Theorem 3.2. Let $I$ be an arbitrary set of indexes. For each $i \in I$, suppose that $\left(\Omega^{i}, \mathcal{F}^{i}, P^{i},\left\{\mathcal{F}_{t}^{i}\right\}_{t \geq 0}, B^{i}, X^{i}, K^{i}\right)$ is a weak solution of the equation

$$
d X_{t}^{i}+A\left(X_{t}^{i}\right) d t \ni b^{i}\left(t, X^{i}\right) d t+\sigma^{i}\left(t, X^{i}\right) d B_{t}^{i}, \quad t \in[0, T],
$$

where the progressively measurable processes $b^{i}:[0, T] \times \mathbf{W} \rightarrow \mathbb{R}^{d}$ and $\sigma^{i}:[0, T] \times$ $\mathbf{W} \rightarrow \mathbb{R}^{d \times d^{\prime}}$ satisfy (3.1) with the same $\alpha>0$, and $\sup _{i \in I} \mathbb{E}^{i}\left|X_{0}^{i}\right|^{2}<+\infty$. Then $\left(X^{i}, K^{i}\right)_{i \in I}$ is tight in $C\left([0, T] ; \mathbb{R}^{d}\right) \times C\left([0, T] ; \mathbb{R}^{d}\right)$.

Proof. We will denote by $C$ the different constants which do not depend on $i, \delta, a$ and $\rho$. Of course, as in the proof of Proposition 2.2, we can suppose that $0 \in$ $\operatorname{Int}(\operatorname{Dom} A)$ and $0 \in A(0)$.

Let us consider, for $\varepsilon>0$ and $a>0$, the set

$$
\mathbb{K}_{\varepsilon, a}:=\left\{x \in \mathbb{R}^{d}|| x-y \mid \geq \varepsilon, \forall y \notin \overline{\operatorname{Dom} A}\right\} \cap \overline{B(0 ; a)} .
$$

Then $\mathbb{K}_{\varepsilon, a}$ is a convex compact set included in $\operatorname{Int}(\operatorname{Dom} A)$, and is nonempty if $\varepsilon$ is smaller than a certain $\varepsilon_{0}>0$ (independent of $a$ ).

Let $f_{a}:(0,+\infty) \rightarrow[0,+\infty)$ be the function defined by

$$
f_{a}(\varepsilon):=\sup \left\{\left|x^{*}\right| \mid \exists x \in \mathbb{K}_{\varepsilon, a}: x^{*} \in A(x)\right\}
$$

(by convention, $\sup \emptyset=0$ ). Of course, since $A$ is locally bounded on $\operatorname{Int}(\operatorname{Dom} A$ ), this is a well-defined, non-increasing function. Let us put, for $\delta>0$ and $a>0$,

$$
g_{a}(\delta):=\inf \left\{\varepsilon \in\left(0, \varepsilon_{0}\right) \mid f_{a}(\varepsilon) \leq \delta^{-1 / 2}\right\}
$$

(by convention, $\left.\inf \emptyset=\varepsilon_{0}\right)$. Then $f_{a}\left(\delta+g_{a}(\delta)\right) \leq \delta^{-1 / 2}$ for every $\delta>0$. We also have $\lim _{\delta \backslash 0} g_{a}(\delta)=0$; let $\delta_{a}>0$ such that $\delta_{a}+g_{a}\left(\delta_{a}\right)<\varepsilon_{0}$.

We estimate now the modulus of continuity of $X^{i}, i \in I$. First of all, by Proposition 2.2, we can find a constant $C$ such that

$$
\mathbb{E}^{i}\left\|X^{i}\right\|_{\mathbf{W}}^{2}+\mathbb{E}^{i}\left\|K^{i}\right\|_{\mathrm{BV}} \leq C\left(1+\mathbb{E}^{i}\left|X_{0}^{i}\right|^{2}\right), \quad \forall i \in I .
$$

Let $s \in[0, T[$ be fixed, but arbitrary. Itô formula for

$$
X_{t}^{i}-X_{s}^{i}=K_{s}^{i}-K_{t}^{i}+\int_{s}^{t} b\left(r, X^{i}\right) d r+\int_{s}^{t} \sigma\left(r, X^{i}\right) d B_{r}^{i}, \quad t \in[s, T]
$$

gives

$$
\begin{aligned}
\left|X_{t}^{i}-X_{s}^{i}\right|^{2}= & 2 \int_{s}^{t}\left\langle X_{s}^{i}-X_{r}^{i}, d K_{r}^{i}\right\rangle+2 \int_{s}^{t}\left\langle X_{r}^{i}-X_{s}^{i}, b\left(r, X^{i}\right)\right\rangle d r \\
& +\int_{s}^{t}\left|\sigma\left(r, X^{i}\right)\right|^{2} d r+2 \int_{s}^{t}\left\langle\left(X_{r}^{i}-X_{s}^{i}\right), \sigma\left(r, X^{i}\right) d B_{r}^{i}\right\rangle .
\end{aligned}
$$


We fix $a>0$ and $\delta \in\left(0, \delta_{a} \wedge 1\right]$. Then $\mathbb{K}_{\delta+g_{a}(\delta), a}$ is nonempty, hence there exists the projection of $X_{s}^{i}$ on $\mathbb{K}_{\delta+g_{a}(\delta), a}$, denoted $Y_{s}^{i, \delta, a}$; therefore we have

$$
\left|X_{s}^{i}-Y_{s}^{i, \delta, a}\right| \leq \delta+g_{a}(\delta), \quad P^{i} \text {-a.s. on } \quad\left\{\left\|X^{i}\right\|_{\mathbf{w}} \leq a\right\},
$$

since $X_{s}^{i} \in \overline{\operatorname{Dom} A}$. Using (2.1)-v) for $\left(X^{i}, K^{i}\right)$ with $z=Y_{s}^{i, \delta, a}, z^{*} \in A(z)$ $\left(A\left(Y_{s}^{i, \delta, a}\right)\right.$ is nonempty!), we obtain

$$
\begin{aligned}
\int_{s}^{t}\left\langle X_{s}^{i}-X_{r}^{i}, d K_{r}^{i}\right\rangle & \leq \int_{s}^{t}\left\langle X_{s}^{i}-Y_{s}^{i, \delta, a}, d K_{r}^{i}\right\rangle+\int_{s}^{t}\left\langle Y_{s}^{i, \delta, a}-X_{r}^{i}, d K_{r}^{i}\right\rangle \\
& \leq\left(\delta+g_{a}(\delta)\right)\left\|K^{i}\right\|_{\mathbf{B V}}+\int_{s}^{t}\left\langle Y_{s}^{i, \delta, a}-X_{r}^{i}, z^{*}\right\rangle d r \\
& \leq\left(\delta+g_{a}(\delta)\right)\left\|K^{i}\right\|_{\mathbf{B V}}+2(t-s) a \cdot f_{a}\left(\delta+g_{a}(\delta)\right) \\
& \leq\left(\delta+g_{a}(\delta)\right)\left\|K^{i}\right\|_{\mathbf{B V}}+2 \delta^{1 / 2} a,
\end{aligned}
$$

$P^{i}$-a.s. on $\left\{\left\|X^{i}\right\|_{\mathbf{w}} \leq a\right\}$, if $0 \leq t-s \leq \delta$.

By (2.1)-v), the assumptions on $b^{i}$ and $\sigma^{i}$, and (3.3) we conclude that, for $0 \leq t-s \leq \delta$ :

$$
\begin{aligned}
\left|X_{t}^{i}-X_{s}^{i}\right|^{2} \mathbf{1}_{\left\{\left\|X^{i}\right\|_{\mathbf{W}} \leq a\right\}} \leq & \left(\delta+g_{a}(\delta)\right)\left\|K^{i}\right\|_{\mathbf{B V}}+2 \delta^{1 / 2} a+4 \alpha \delta a(1+a) \\
& +2 \alpha^{2} \delta\left(1+a^{2}\right)+2 a\left|\int_{s}^{t} \sigma\left(r, X^{i}\right) d B_{r}^{i}\right| \\
& +2\left|\int_{s}^{t}\left\langle X_{r}^{i}, \sigma\left(r, X^{i}\right) d B_{r}^{i}\right\rangle\right| .
\end{aligned}
$$

Hence

$$
\begin{aligned}
\sup _{0 \leq t-s \leq \delta}\left|X_{t}^{i}-X_{s}^{i}\right|^{2} \mathbf{1}_{\left\{\left\|X^{i}\right\|_{\mathbf{W}} \leq a\right\} \leq} & \left(\delta+g_{a}(\delta)\right)\left\|K^{i}\right\|_{\mathbf{B V}}+C \delta^{1 / 2}\left(1+a^{2}\right) \\
& +2 a \sup _{0 \leq t-s \leq \delta}\left|\int_{s}^{t} \sigma\left(r, X^{i}\right) d B_{r}^{i}\right| \\
& +2 \sup _{0 \leq t-s \leq \delta}\left|\int_{s}^{t}\left\langle X_{r}^{i}, \sigma\left(r, X^{i}\right) d B_{r}^{i}\right\rangle\right| .
\end{aligned}
$$

Let us consider $\rho>0$. Then, for each $\delta>0$ and $a>0$, we have

$$
\begin{aligned}
\left.P^{i} \sup _{0 \leq t-s \leq \delta}\left|X_{t}^{i}-X_{s}^{i}\right| \geq \rho\right] \leq & P^{i}\left[\sup _{0 \leq t-s \leq \delta}\left|X_{t}^{i}-X_{s}^{i}\right| \geq \rho ;\left\|X^{i}\right\|_{\mathbf{w}} \leq a\right] \\
& +P^{i}\left[\left\|X^{i}\right\|_{\mathbf{W}}>a\right] \\
\leq & P^{i}\left[\sup _{0 \leq t-s \leq \delta}\left|X_{t}^{i}-X_{s}^{i}\right|^{2} \mathbf{1}_{\left\{\left\|X^{i}\right\|_{\mathbf{w}} \leq a\right\}} \geq \rho^{2}\right] \\
& +\frac{\mathbb{E}^{i}\left\|X^{i}\right\|_{\mathbf{W}}^{2}}{a^{2}} .
\end{aligned}
$$


From (3.2) and (3.4) we obtain that, for every $i \in I$ :

$$
\begin{aligned}
\left.P^{i} \sup _{0 \leq t-s \leq \delta}\left|X_{t}^{i}-X_{s}^{i}\right| \geq \rho\right] \leq & P^{i}\left[\left(\delta+g_{a}(\delta)\right)\left\|K^{i}\right\|_{\mathrm{BV}}+C \delta^{1 / 2}\left(1+a^{2}\right) \geq \frac{\rho^{2}}{3}\right] \\
& +P^{i}\left[\sup _{0 \leq t-s \leq \delta}\left|\int_{s}^{t}\left\langle X_{r}^{i}, \sigma\left(r, X^{i}\right) d B_{r}^{i}\right\rangle\right| \geq \frac{\rho^{2}}{6}\right] \\
& +P^{i}\left[\sup _{0 \leq t-s \leq \delta}\left|\int_{s}^{t} \sigma\left(r, X^{i}\right) d B_{r}^{i}\right| \geq \frac{\rho^{2}}{6 a}\right] \\
& +\frac{C}{a^{2}} .
\end{aligned}
$$

We recall now that if $\left(M^{i}\right)_{i \in I}$ is a family of continuous local martingales such that $\left(M_{0}^{i}\right)_{i \in I}$ is uniformly bounded in probability and $\left(\left\langle M^{i}\right\rangle\right)_{i \in I}$ is tight, then $\left(M^{i}\right)_{i \in I}$ is tight [18, Lemma 1]. This allows us to conclude that the processes $\left(\int_{0}^{\cdot}\left\langle X_{s}^{i}, \sigma\left(s, X^{i}\right) d B_{s}^{i}\right\rangle\right)_{i \in I}$ and $\left(\int_{0}^{\cdot} \sigma\left(s, X^{i}\right) d B_{s}^{i}\right)_{i \in I}$ are tight in $C([0, T])$, respectively in $C\left([0, T] ; \mathbb{R}^{d}\right)$. Indeed, from (3.1), (3.2), and Proposition 3.1 it follows that $\left(\int_{0}^{\cdot}\left|\left(X_{s}^{i}\right)^{*} \sigma\left(s, X^{i}\right)\right|^{2} d s\right)_{i \in I}$ and $\left(\int_{0}^{\cdot}\left|\sigma\left(s, X^{i}\right)\right|^{2} d s\right)_{i \in I}$ are tight (here * denotes the transposition of a matrix).

From (3.5), (3.2), and the "only if" part of Proposition 3.1 we obtain

$$
\varlimsup_{\delta \searrow 0} \sup _{i \in I} P^{i}\left[\sup _{0 \leq t-s \leq \delta}\left|X_{t}^{i}-X_{s}^{i}\right| \geq \rho\right] \leq \frac{C}{a^{2}}, \quad \forall a>0 ;
$$

hence

$$
\lim _{\delta \searrow 0} \sup _{i \in I} P^{i}\left[\sup _{0 \leq t-s \leq \delta}\left|X_{t}^{i}-X_{s}^{i}\right| \geq \rho\right]=0 .
$$

On the other hand, it is easily seen that

$$
\lim _{a \nearrow \infty} \sup _{i \in I} P^{i}\left[\left|X_{0}^{i}\right| \geq a\right]=0 .
$$

According to the "if" part of Proposition 3.1, $\left(X^{i}\right)_{i \in I}$ is then tight in $C\left([0, T] ; \mathbb{R}^{d}\right)$.

In order to accomplish the proof, we observe that the families of processes $\left(\int_{0}^{\cdot} b\left(s, X^{i}\right) d s\right)_{i \in I}$ and $\left(\int_{0}^{\cdot} \sigma\left(s, X^{i}\right) d B_{s}^{i}\right)_{i \in I}$ are tight. Since

$$
K_{t}^{i}=X_{0}^{i}-X_{t}^{i}+\int_{0}^{t} b\left(s, X^{i}\right) d s+\int_{0}^{t} \sigma\left(s, X^{i}\right) d B_{s}^{i}, \forall t \in[0, T], P^{i} \text { a.s. },
$$

it follows that the family $\left(K^{i}\right)_{i \in I}$ is also tight.

This result is an important step towards the construction (as a limit obtained by a compactness argument) of the law of the solution we are searching for. Therefore, we will work on the canonical space $\mathbf{W} \times \mathbf{W}$, and follow the "martingale problem" approach, which has the advantage of using only the image laws of the solutions, without (apparently) taking into account the Brownian motion. 
We start by introducing some useful notations:

$$
\begin{aligned}
\bar{\Omega} & :=\mathbf{W} \times \mathbf{W}, \quad \overline{\mathcal{F}}:=\mathcal{B}(\mathbf{W}) \otimes \mathcal{B}(\mathbf{W})=\mathcal{B}(\bar{\Omega}) ; \\
\overline{\mathcal{F}}_{t} & :=\mathcal{B}_{t} \otimes \mathcal{B}_{t}, \quad t \geq 0 ; \\
\bar{\Omega}_{0} & :=\left\{(x, \eta) \in \bar{\Omega} \mid \eta \in \mathcal{A}(x), \eta_{0}=0\right\} ; \\
F_{a} & :=\left\{(x, \eta) \in \bar{\Omega}_{0} \mid\|\eta\|_{\mathbf{B V}} \leq a\right\}, \quad a \geq 0
\end{aligned}
$$

(see (2.2) for the definition of $\mathcal{A}$ ). The sets $F_{a}, a \geq 0$, are closed in $\bar{\Omega}$ (in consequence $\bar{\Omega}_{0} \in \overline{\mathcal{F}}$ ), as we can immediately deduce from the next lemma:

Lemma 3.3 (Helly-Bray). Let $\left(\eta_{n}\right)_{n \geq 1}$ be a sequence in $\mathbf{W} \cap B V\left([0, T] ; \mathbb{R}^{d}\right)$ which converges (punctually) to a function $\eta \in \mathbf{W}$. If $\sup _{n \geq 1}\left\|\eta_{n}\right\|_{\mathbf{B V}}<+\infty$, then $\eta \in$ $B V\left([0, T] ; \mathbb{R}^{d}\right)$ and

$$
\lim _{n \rightarrow \infty} \int_{0}^{T}\left\langle x(r), d \eta_{n}(r)\right\rangle=\int_{0}^{T}\langle x(r), d \eta(r)\rangle,
$$

for every $x \in \mathbf{W}$ (i.e. $\eta_{n} \stackrel{w^{*}}{\longrightarrow} \eta$ ).

If $R$ is a probability measure on $(\bar{\Omega}, \overline{\mathcal{F}})$ such that $R\left(\bar{\Omega}_{0}\right)=1$, let us denote $\mathcal{N}_{R}$ the family of $R$-negligible sets in $\overline{\mathcal{F}}$ and $\overline{\mathcal{F}}^{R}:=\overline{\mathcal{F}} \vee \mathcal{N}_{R}, \overline{\mathcal{F}}_{t}^{R}:=\bigcap_{s>t}\left(\overline{\mathcal{F}}_{s} \vee \mathcal{N}_{R}\right)$ for $t \geq 0$. It is clear now that $\left(\bar{\Omega}, \overline{\mathcal{F}}^{R}, R,\left\{\overline{\mathcal{F}}_{t}^{R}\right\}_{t>0}\right)$ is a stochastic basis and $\bar{\Omega}_{0} \in \overline{\mathcal{F}}_{0}^{R}$. Hence the canonical processes $\bar{X}: \bar{\Omega} \rightarrow \mathbf{W}, \bar{K}: \bar{\Omega} \rightarrow \mathbf{W}$, defined by

$$
\bar{X}(x, \eta):=x, \quad \bar{K}(x, \eta):= \begin{cases}\eta, & \text { if } \quad(x, \eta) \in \bar{\Omega}_{0} ; \\ 0, & \text { if } \quad(x, \eta) \in \bar{\Omega} \backslash \bar{\Omega}_{0},\end{cases}
$$

are progressively measurable with respect to this basis.

The next result, which will be used in the sequel and which is interesting by itself, is a generalization of Lemma 3.3 in the stochastic framework.

Proposition 3.4. Let $X^{n}, K^{n}:\left(\Omega^{n}, \mathcal{F}^{n}, P^{n}\right) \rightarrow \mathbf{W}, n \geq 1$, be two sequences of random variables, converging in distribution to $X$, respectively $K$. If, for $n \geq 1$, $K^{n}$ is a.s. with bounded variation and

$$
\lim _{a \rightarrow+\infty} \sup _{n \geq 1} P^{n}\left(\left\|K^{n}\right\|_{\mathrm{BV}}>a\right)=0,
$$

then $K$ is a.s. with bounded variation and $\int_{0}^{T}\left\langle X_{t}, d K_{t}\right\rangle$ is the limit in distribution of the sequence $\left(\int_{0}^{T}\left\langle X_{t}^{n}, d K_{t}^{n}\right\rangle\right)$.

Proof. Let us put

$$
\Lambda(x, \eta):=\int_{0}^{T}\left\langle\bar{X}_{t}(x, \eta), d \bar{K}_{t}(x, \eta)\right\rangle, \quad(x, \eta) \in \bar{\Omega},
$$

where $\bar{X}$ and $\bar{K}$ are the processes defined in (3.7). According to Lemma 3.3, the set

$$
G_{a}:=\left\{(x, \eta) \in \bar{\Omega} \mid\|\eta\|_{\mathbf{B V}} \leq a\right\}
$$


is closed and the restriction of $\Lambda$ to $G_{a}$ is continuous, for every $a>0$. Therefore we can extend this restriction to a function $\Lambda_{a}$, continuous on $G_{a}$.

If we denote by $R_{n}$ the distribution of $\left(X^{n}, K^{n}\right)$ and by $R_{0}$ the distribution of $(X, K)$, then the condition of the statement is equivalent to

$$
\lim _{a \rightarrow+\infty} \sup _{n \geq 1} R_{n}\left(\bar{\Omega} \backslash G_{a}\right)=0 .
$$

Consequently, the weak convergence of $R_{n}$ to $R_{0}$ implies that $\lim _{a \rightarrow+\infty} R_{0}\left(G_{a}\right)=1$, hence $R_{0}\left(\bigcup_{a>0} G_{a}\right)=1$, i.e. $K$ is a.s. with bounded variation.

Now, let $f: \mathbb{R} \rightarrow \mathbb{R}$ be a continuous bounded function. Then $\left(\mathbb{E}_{R_{n}}\left(f \circ \Lambda_{a}\right)\right)$ converges to $\mathbb{E}_{R_{0}}\left(f \circ \Lambda_{a}\right)$, for every $a>0$.

On the other hand, for all $n \geq 0$ and $a>0$,

$$
\begin{aligned}
\left|\mathbb{E}_{R_{n}}\left(f \circ \Lambda_{a}-f \circ \Lambda\right)\right| & =\left|\mathbb{E}_{R_{n}}\left(f \circ \Lambda_{a}-f \circ \Lambda\right) \mathbf{1}_{\bar{\Omega} \backslash G_{a}}\right| \\
& \leq 2 \sup _{x \in \mathbb{R}}|f(x)| \cdot R_{n}\left(\bar{\Omega} \backslash G_{a}\right) .
\end{aligned}
$$

From this relation and (3.8) we deduce that $\left(\mathbb{E}_{R_{n}}(f \circ \Lambda)\right)$ converges to $\mathbb{E}_{R_{0}}(f \circ \Lambda)$. The conclusion of the proposition is derived from the fact that the distributions of $\int_{0}^{T}\left\langle X_{t}^{n}, d K_{t}^{n}\right\rangle$ and $\int_{0}^{T}\left\langle X_{t}, d K_{t}\right\rangle$ coincide with $R_{n} \circ \Lambda^{-1}$, respectively $R_{0} \circ \Lambda^{-1}$.

Let us consider the differential operator defined by

$\mathcal{L} f(t, x):=\frac{1}{2} \operatorname{tr} \sigma \sigma^{*}(t, x) D^{2} f(x(t))+\langle b(t, x), D f(x(t))\rangle, \quad(t, x) \in[0, T] \times \mathbf{W}$, for $f \in C^{2}\left(\mathbb{R}^{d}\right)\left(D:=\left(\frac{\partial}{\partial x_{1}}, \ldots, \frac{\partial}{\partial x_{d}}\right), D^{2}:=\left(\frac{\partial^{2}}{\partial x_{i} \partial x_{j}}\right)_{1 \leq i, j \leq d}\right)$.

Definition. We say that a probability measure $R$ on $\bar{\Omega}$ is a solution of the martingale problem for (1.1) if $R\left(\bar{\Omega}_{0}\right)=1$ and for each $f \in C_{c}^{2}\left(\mathbb{R}^{d}\right)^{\dagger}$, the continuous process

$$
\bar{M}_{t}^{f}:=f\left(\bar{X}_{t}\right)-f\left(\bar{X}_{0}\right)-\int_{0}^{t} \mathcal{L} f(s, \bar{X}) d s+\int_{0}^{t}\left\langle D f\left(\bar{X}_{s}\right), d \bar{K}_{s}\right\rangle, \quad t \in[0, T],
$$

is a martingale under $\left(\bar{\Omega}, \overline{\mathcal{F}}^{R}, R,\left\{\overline{\mathcal{F}}_{t}^{R}\right\}_{t \geq 0}\right)$.

Remark. In order to ensure the existence of the above integrals, one should impose the condition

$$
\int_{0}^{T}\left(|b(t, \bar{X})|+|\sigma(t, \bar{X})|^{2}\right) d t<+\infty, \quad R \text {-a.s. }
$$

which is, of course, implied by (3.1).

Let us now see which is the connection between the martingale problem and weak solutions.

Definition. Let $\left(\Omega, \mathcal{F}, P,\left\{\mathcal{F}_{t}\right\}_{t \geq 0}\right)$ and $\left(\hat{\Omega}, \hat{\mathcal{F}}, \hat{P},\left\{\hat{\mathcal{F}}_{t}\right\}_{t \geq 0}\right)$ be two stochastic basis. We say that the last one is an extension of the first if there exists a random variable $\theta:(\hat{\Omega}, \hat{\mathcal{F}}) \rightarrow(\Omega, \mathcal{F})$, called projection, such that:

${ }^{\dagger} C_{c}^{2}\left(\mathbb{R}^{d}\right)$ denotes the space of functions in $C^{2}\left(\mathbb{R}^{d}\right)$ with compact support. 
i) $P=\hat{P} \circ \theta^{-1}$;

ii) for any $Y \in L^{1}(\Omega, \mathcal{F}, P)$ and $t \geq 0, \hat{\mathbb{E}}\left(Y \circ \theta \mid \hat{\mathcal{F}}_{t}\right)=\mathbb{E}\left(Y \mid \mathcal{F}_{t}\right) \circ \theta, \hat{P}$-a.s.

Remark. Let $\left(\hat{\Omega}, \hat{\mathcal{F}}, \hat{P},\left\{\hat{\mathcal{F}}_{t}\right\}_{t \geq 0}\right)$ be an extension of $\left(\Omega, \mathcal{F}, P,\left\{\mathcal{F}_{t}\right\}_{t \geq 0}\right)$ with projection $\theta$. Then $\theta$ is $\hat{\mathcal{F}}_{t} / \mathcal{F}_{t}$-measurable for every $t \geq 0$. Moreover, a stochastic process $M$ is a martingale with respect to the first stochastic basis if and only if $M \circ \theta$ is a martingale with respect to its extension.

Proposition 3.5. Let $R$ be a solution of the martingale problem. Then there exists an extension $\left(\Omega, \mathcal{F}, P,\left\{\mathcal{F}_{t}\right\}_{t>0}\right)$ of $\left(\bar{\Omega}, \overline{\mathcal{F}}^{R}, R,\left\{\overline{\mathcal{F}}_{t}^{R}\right\}_{t>0}\right)$ with projection $\theta$ and a standard, $d^{\prime}$-dimensional Brownian motion $B$ with respect to this extension such that $\left(\Omega, \mathcal{F}, P,\left\{\mathcal{F}_{t}\right\}_{t>0}, B, X, K\right)$ is a weak solution of (1.1), where $X:=\bar{X} \circ \theta$, $K:=\bar{K} \circ \theta$.

Conversely, if (3.1) holds and $\left(\Omega, \mathcal{F}, P,\left\{\mathcal{F}_{t}\right\}_{t \geq 0}, B, X, K\right)$ is a weak solution of (1.1) with $\mathbb{E}\left|X_{0}\right|^{2}<+\infty$, then $P \circ(X, K)^{-1}$ is a solution of the martingale problem.

Proof. We will give only a sketch of the proof, because of its similitude with that of the univalued case (see, for example, [12, Proposition 5.4.6]).

Suppose that $R$ is a solution of the martingale problem. Then, for the choices $f(x)=x_{j}, f(x)=x_{j} x_{k}, 1 \leq j, k \leq d\left(x_{j}\right.$ denotes the $j^{\text {th }}$ component of $\left.x\right)$, the processes

$$
\begin{aligned}
\bar{M}_{j, t}: & =\bar{X}_{j, t}-\bar{X}_{j, 0}-\int_{0}^{t} b_{j}(s, \bar{X}) d s+\bar{K}_{j, t}, \quad t \in[0, T] \\
\bar{M}_{j, k, t}:= & \bar{X}_{j, t} \bar{X}_{k, t}-\bar{X}_{j, 0} \bar{X}_{k, 0}+\int_{0}^{t} \bar{X}_{j, s} d \bar{K}_{k, s}+\int_{0}^{t} \bar{X}_{k, s} d \bar{K}_{j, s} \\
& -\int_{0}^{t}\left[a_{j k}(s, \bar{X})+\bar{X}_{j, s} b_{k}(s, \bar{X})+\bar{X}_{k, s} b_{j}(s, \bar{X})\right] d s, \quad t \in[0, T],
\end{aligned}
$$

are continuous local martingales, where $a:=\sigma \sigma^{*}$. Indeed, we can approximate $f$ by functions in $C_{c}^{2}\left(\mathbb{R}^{d}\right)$, the result being accomplished by localization.

Applying the Itô formula to $\bar{X}_{t}^{j} \bar{X}_{t}^{k}$ we obtain

$$
\bar{M}_{j, k, t}=\left\langle\bar{M}_{j}, \bar{M}_{k}\right\rangle_{t}-\int_{0}^{t} a_{j k}(s, \bar{X}) d s, \quad t \in[0, T] .
$$

But a continuous local martingale has bounded variation if and only if it is the null process (see, for instance [12, Exercise 1.5.21]). Hence

$$
\left\langle\bar{M}_{j}, \bar{M}_{k}\right\rangle_{t}=\int_{0}^{t} a_{j k}(s, \bar{X}) d s, \quad \forall t \in[0, T], \quad R \text {-a.s. }
$$

Exactly as in the proof of [12, Proposition 5.4.6], we can find an extension $\left(\Omega, \mathcal{F}, P,\left\{\mathcal{F}_{t}\right\}_{t \geq 0}\right)$ of $\left(\bar{\Omega}, \overline{\mathcal{F}}^{R}, R,\left\{\overline{\mathcal{F}}_{t}^{R}\right\}_{t \geq 0}\right.$ ) (with projection $\left.\theta\right)$ and an $\left\{\mathcal{F}_{t}\right\}_{t \geq 0^{-}}$ adapted standard, $d^{\prime}$-dimensional Brownian motion $B$ such that, with the notation 


$$
\begin{aligned}
M_{j, t}:=\bar{M}_{j, t} \circ \theta, X & :=\bar{X} \circ \theta, K:=\bar{K} \circ \theta, \\
M_{j, t} & =\int_{0}^{t} \sigma_{j}(s, X) d B_{s}, \quad \forall t \in[0, T], \quad P \text {-a.s. },
\end{aligned}
$$

for every $1 \leq j \leq d$. From (3.9) and the fact that $R\left(\bar{\Omega}_{0}\right)=1$ it follows easily that $\left(\Omega, \mathcal{F}, P,\left\{\mathcal{F}_{t}\right\}_{t \geq 0}, B, X, K\right)$ is a weak solution of (1.1).

For the converse, let $\left(\Omega, \mathcal{F}, P,\left\{\mathcal{F}_{t}\right\}_{t>0}, B, X, K\right)$ be a weak solution of (1.1) with $\mathbb{E}\left|X_{0}\right|^{2}<+\infty$. Then, applying the Itô formula to the process $f\left(X_{t}\right)$, where $f \in C_{c}^{2}\left(\mathbb{R}^{d}\right)$, we see that

$$
\begin{aligned}
M_{t}^{f} & :=f\left(X_{t}\right)-f\left(X_{0}\right)-\int_{0}^{t} \mathcal{L} f(s, X) d s+\int_{0}^{t}\left\langle D f\left(X_{s}\right), d K_{s}\right\rangle \\
& =\int_{0}^{t}\left\langle D f\left(X_{s}\right), \sigma(s, X) d B_{s}\right\rangle, \quad t \in[0, T],
\end{aligned}
$$

is a martingale, since (3.1) holds and $\mathbb{E}\|X\|_{\mathbf{W}}^{2}<+\infty$, cf. Proposition 2.2. Letting $R:=P \circ(X, K)^{-1}$, one can easily show that $\bar{M}_{t}^{f}$ is a martingale with respect to $\left(\bar{\Omega}, \overline{\mathcal{F}}^{R}, R,\left\{\overline{\mathcal{F}}_{t}^{R}\right\}_{t \geq 0}\right)$. From (2.1)-v) it also follows that $R\left(\bar{\Omega}_{0}\right)=1$.

We are now able to prove the main result of the section.

Theorem 3.6. Suppose that $(3.1)$ holds. If $b(t, \cdot)$ and $\sigma(t, \cdot)$ are continuous for almost all $t \in[0, T]$ and $\mu$ is a probability measure on $\overline{\operatorname{Dom} A}$ such that $\int|x|^{2} \mu(d x)<$ $+\infty$, then there exists a weak solution of equation (1.1) with $\mu$ as initial distribution.

Proof. For every $n \in \mathbb{N}^{*}$, we let $b_{n}:[0, T] \times \mathbf{W} \rightarrow \mathbb{R}^{d}$ and $\sigma_{n}:[0, T] \times \mathbf{W} \rightarrow \mathbb{R}^{d \times d^{\prime}}$ be defined by

$$
\begin{aligned}
b_{n}(t, x) & :=b\left(t, x\left(\cdot \wedge \frac{k T}{2^{n}}\right)\right), \\
\sigma_{n}(t, x) & :=\sigma\left(t, x\left(\cdot \wedge \frac{k T}{2^{n}}\right)\right), \quad \text { if } \quad 0 \leq k \leq 2^{n}, \quad(t, x) \in\left[\frac{k T}{2^{n}}, \frac{(k+1) T}{2^{n}}\right) \times \mathbf{W} .
\end{aligned}
$$

The functionals $b_{n}$ and $\sigma_{n}$ are progressively measurable and satisfy (3.1) uniformly in $n \in \mathbb{N}^{*}$. Also $b_{n}(t, x) \rightarrow b(t, x), \sigma_{n}(t, x) \rightarrow \sigma(t, x)$, as $n \rightarrow \infty$, uniformly on compacts of $\mathbf{W}$, for almost all $t \in[0, T]$. We can find a stochastic basis $\left(\Omega, \mathcal{F}, P,\left\{\mathcal{F}_{t}\right\}_{t \geq 0}\right)$, a standard, $d^{\prime}$-dimensional Brownian $B$ with respect to this basis, and a $\mathcal{F}_{0}$-measurable $d$-dimensional random vector $\xi$ such that $P \circ \xi^{-1}=\mu$. By recurrence on $k \in\left\{0,1, \ldots, 2^{n}-1\right\}$, one can prove that there exists a unique strong solution $\left(X^{n}, K^{n}\right)$ of the equation

$$
\left\{\begin{array}{l}
d X_{t}^{n}+A\left(X_{t}^{n}\right) d t \ni b_{n}\left(t, X^{n}\right) d t+\sigma_{n}\left(t, X^{n}\right) d B_{t}, \quad t \in[0, T] \\
X_{0}^{n}=\xi,
\end{array}\right.
$$

for every $n \in \mathbb{N}^{*}$, since on every interval $\left[\frac{k T}{2^{n}}, \frac{(k+1) T}{2^{n}}\right], b_{n}$ and $\sigma_{n}$ do not depend on the state variable and therefore we can apply Theorem 2.3 . 
Let $R_{n}:=P \circ\left(X^{n}, K^{n}\right)^{-1}$. By Theorem 3.2 and Prohorov's theorem, the sequence $\left\{R_{n}\right\}_{n \in \mathbb{N}^{*}}$ is relatively compact in $\mathcal{P}(\bar{\Omega})$. Hence we can extract a subsequence, which, without loss of generality, we still denote $\left(R_{n}\right)$, converging to a probability measure $R_{0} \in \mathcal{P}(\bar{\Omega})$. Let us prove that $R_{0}$ is a solution of the martingale problem for (1.1)

First we notice that, by Proposition 2.2, there exists $C>0$ such that

$$
\mathbb{E}_{R_{n}}\|\bar{X}\|_{\mathbf{W}}^{2}+\mathbb{E}_{R_{n}}\|\bar{K}\|_{\mathbf{B V}} \leq C\left(1+\int|x|^{2} \mu(d x)\right), \quad \forall n \in \mathbb{N}^{*} .
$$

We show first that $R_{0}\left(\bar{\Omega}_{0}\right)=1$. Indeed, $F_{a}$ (see $(3.6)$ for the definition) is closed for every $a \geq 0$, hence

$$
\begin{aligned}
R_{0}\left(\bar{\Omega}_{0}\right) & \geq R_{0}\left(F_{a}\right) \geq \limsup _{n \rightarrow \infty} R_{n}\left(F_{a}\right)=1-\liminf _{n \rightarrow \infty} R_{n}\left(\|\bar{K}\|_{\mathbf{B V}}>a\right) \\
& \geq 1-\frac{1}{a} \liminf _{n \rightarrow \infty} \mathbb{E}_{R_{n}}\|\bar{K}\|_{\mathbf{B V}} \geq 1-\frac{C}{a}\left(1+\int|x|^{2} \mu(d x)\right) .
\end{aligned}
$$

Let, for $n \in \mathbb{N}, f \in C_{c}^{2}\left(\mathbb{R}^{d}\right)$,

$$
\bar{M}_{t}^{f, n}:=f\left(\bar{X}_{t}\right)-f\left(\bar{X}_{0}\right)-\int_{0}^{t} \mathcal{L}^{n} f(s, \bar{X}) d s+\int_{0}^{t}\left\langle D f\left(\bar{X}_{s}\right), d \bar{K}_{s}\right\rangle, \quad t \in[0, T],
$$

where $\mathcal{L}^{n} f:=\frac{1}{2} \operatorname{tr} \sigma_{n} \sigma_{n}^{*} D^{2} f+\left\langle b_{n}, D f\right\rangle$. Here $b_{0}$ and $\sigma_{0}$ stand for $b$, respectively for $\sigma$, in order to unify the notations.

We observe that, for a probability measure $R$ on $\bar{\Omega}$, an integrable, $\left\{\overline{\mathcal{F}}_{t}^{R}\right\}_{t \geq 0^{-}}$ adapted process $M$ is a martingale under $\left(\bar{\Omega}, \overline{\mathcal{F}}^{R}, R,\left\{\overline{\mathcal{F}}_{t}^{R}\right\}_{t \geq 0}\right)$ if and only if $\mathbb{E}_{R}\left(M_{t}-M_{s}\right) \Gamma=0$ for each $0 \leq s<t \leq T$ and for every bounded, real-valued, continuous, $\overline{\mathcal{F}}_{s}$-measurable function $\Gamma$.

Let us fix $f \in C_{c}^{2}\left(\mathbb{R}^{d}\right), \Gamma$ as above, and $s, t \in[0, T]$ such that $s<t$. Then, by Proposition 3.5 we have

$$
\mathbb{E}_{R_{n}}\left(\bar{M}_{t}^{f, n}-\bar{M}_{s}^{f, n}\right) \Gamma=0, \quad \forall n \in \mathbb{N}^{*}
$$

It remains to prove that we can pass to the limit as $n \rightarrow \infty$ in this relation, in order to obtain it not only for $n>0$, but also for $n=0$.

We note that the function $\left(f\left(\bar{X}_{t}\right)-f\left(\bar{X}_{s}\right)\right) \Gamma$ is bounded and continuous within the uniform convergence topology, hence

$$
\lim _{n \rightarrow \infty} \mathbb{E}_{R_{n}}\left(f\left(\bar{X}_{t}\right)-f\left(\bar{X}_{s}\right)\right) \Gamma=\mathbb{E}_{R_{0}}\left(f\left(\bar{X}_{t}\right)-f\left(\bar{X}_{s}\right)\right) \Gamma .
$$

On the other hand, since $\left(\mathcal{L}^{n} f(r, \bar{X})\right)_{n \geq 1}$ converges uniformly on compacts of $\bar{\Omega}$ to $\mathcal{L}^{0} f(r, \bar{X})$ for almost all $r \in[0, T]$ and since $\left(R_{n}\right)$ converges to $R_{0}$, we have

$$
\lim _{n \rightarrow \infty} \mathbb{E}_{R_{n}} \mathcal{L}^{n} f(r, \bar{X})=\mathbb{E}_{R_{0}} \mathcal{L}^{0} f(r, \bar{X}) \quad \text { for almost all } \quad r \in[0, T] .
$$


From (3.12), Lebesgue's dominated convergence theorem, and Fubini's theorem, it follows that

$$
\begin{aligned}
\lim _{n \rightarrow \infty} \mathbb{E}_{R_{n}}\left[\left(f\left(\bar{X}_{t}\right)-\right.\right. & \left.\left.f\left(\bar{X}_{s}\right)-\int_{s}^{t} \mathcal{L}^{n} f(r, \bar{X}) d r\right) \Gamma\right] \\
= & \mathbb{E}_{R_{0}}\left[\left(f\left(\bar{X}_{t}\right)-f\left(\bar{X}_{s}\right)-\int_{s}^{t} \mathcal{L}^{0} f(r, \bar{X}) d r\right) \Gamma\right] .
\end{aligned}
$$

The function $\int_{s}^{t}\left\langle D f\left(\bar{X}_{s}\right), d \bar{K}_{s}\right\rangle$ is not necessarily continuous on $\bar{\Omega}$, but we can apply Proposition 3.4 (with interval $[s, t]$ instead of $[0, T]$ ) and conclude that

$$
\lim _{n \rightarrow \infty} \mathbb{E}_{R_{n}}\left[\Gamma \int_{s}^{t}\left\langle D f\left(\bar{X}_{r}\right), d \bar{K}_{r}\right\rangle\right]=\mathbb{E}_{R_{0}}\left[\Gamma \int_{s}^{t}\left\langle D f\left(\bar{X}_{r}\right), d \bar{K}_{r}\right\rangle\right] .
$$

Indeed, $\Gamma D f\left(\bar{X}_{s}\right)$ (under $\left(\bar{\Omega}, \overline{\mathcal{F}}, R_{n}\right)$ ), converges in distribution to $\Gamma D f\left(\bar{X}_{s}\right)$ (under $\left.\left(\bar{\Omega}, \overline{\mathcal{F}}, R_{0}\right)\right)$ and $\lim _{a \rightarrow+\infty} \sup _{n \geq 1} R_{n}\left(\left\|K^{n}\right\|_{\mathbf{B V}}>a\right)=0$.

The convergences (3.13) and (3.14) ensure us that we can pass to the limit in (3.11), and hence prove that $\bar{M}^{f, 0}$ (which is the same as $\bar{M}^{f}$ ) is a solution of the martingale problem for (1.1). Obviously, the condition $R_{0} \circ X_{0}^{-1}=\mu$ is satisfied; therefore, applying once again Proposition 3.5, there exists a weak solution of equation (1.1) with $\mu$ as the initial distribution.

\section{The optimal control problem}

Let $U$ be a nonempty compact metric space, $b:[0, T] \times \mathbf{W} \times U \rightarrow \mathbb{R}^{d}, \sigma$ : $[0, T] \times \mathbf{W} \times U \rightarrow \mathbb{R}^{d \times d^{\prime}}$ two progressively measurable processes (with respect to the filtration $\left.\left\{\mathcal{B}_{t} \otimes \mathcal{B}(U)\right\}_{t \geq 0}\right)$, and $\mu$ a probability measure on $\overline{\operatorname{Dom} A}$. Let us consider the equation

$$
\left\{\begin{array}{l}
d X_{t}+A\left(X_{t}\right) d t \ni b\left(t, X, u_{t}\right) d t+\sigma\left(t, X, u_{t}\right) d B_{t}, \quad t \in[0, T] \\
X_{0} \stackrel{\text { law }}{=} \mu
\end{array}\right.
$$

Definition. We say that $\left(\Omega, \mathcal{F}, P,\left\{\mathcal{F}_{t}\right\}_{t \geq 0}, B, X, K, u\right)$ is a control (in the weak sense) for equation (4.1) if $\left(\Omega, \mathcal{F}, P,\left\{\mathcal{F}_{t}\right\}_{t \geq 0}\right)$ is a stochastic basis, $B$ is a standard, $d^{\prime}$-dimensional Brownian motion with respect to this basis, $u:[0, T] \times \Omega \rightarrow U$ is a progressively measurable process, called control policy, and $(X, K)$ is a solution of (4.1) under $\left(\Omega, \mathcal{F}, P,\left\{\mathcal{F}_{t}\right\}_{t \geq 0}\right)$.

As stated at the beginning of the paper, $A$ is assumed to be a maximal monotone operator with $\operatorname{Int}(\operatorname{Dom} A) \neq \emptyset$. We impose the usual growth condition

$$
|b(t, x, v)|+|\sigma(t, x, v)| \leq \alpha\left(1+\|x\|_{\mathbf{W}}\right), \quad \forall(t, x, v) \in[0, T] \times \mathbf{W} \times U .
$$


The coefficients $g:[0, T] \times \mathbf{W} \times U \rightarrow]-\infty,+\infty]$ and $\left.\left.h: \mathbb{R}^{d} \rightarrow\right]-\infty,+\infty\right]$ are measurable functions, which satisfy

i) $g(t, \cdot, v)$ is l.s.c., uniformly with respect to $(t, v) \in[0, T] \times U$;

ii) $g(t, x, \cdot)$ is l.s.c., $\forall(t, x) \in[0, T] \times \mathbf{W}$;

iii) $g(t, x, v) \geq \beta(t), \forall(t, x, v) \in[0, T] \times \mathbf{W} \times U$;

iv) $h$ is l.s.c. and lower bounded,

where $\beta \in L^{1}(0, T)$.

The problem we want to solve is that of minimizing the cost functional

$$
J(\mathcal{U}):=\mathbb{E}\left[\int_{0}^{T} g\left(t, X, u_{t}\right) d t+h\left(X_{T}\right)\right]
$$

over the class of all controls $\mathcal{U}:=\left(\Omega, \mathcal{F}, P,\left\{\mathcal{F}_{t}\right\}_{t \geq 0}, B, X, K, u\right)$.

Some of the results obtained in the previous section can be easily extended to the current framework, so we will state them without proof. The following result corresponds to Theorem 3.2:

Theorem 4.1. Let $I$ be an arbitrary set of indexes. For each $i \in I$, suppose that $\left(\Omega^{i}, \mathcal{F}^{i}, P^{i},\left\{\mathcal{F}_{t}^{i}\right\}_{t \geq 0}, B^{i}, X^{i}, K^{i}, u^{i}\right)$ is a control for the equation

$$
\left\{\begin{array}{l}
d X_{t}^{i}+A\left(X_{t}^{i}\right) d t \ni b_{i}\left(t, X^{i}, u_{t}^{i}\right) d t+\sigma_{i}\left(t, X^{i}, u_{t}^{i}\right) d B_{t}^{i}, \quad t \in[0, T] ; \\
X_{0}^{i} \stackrel{\text { law }}{=} \mu,
\end{array}\right.
$$

where the progressively measurable processes $b^{i}:[0, T] \times \mathbf{W} \times U \rightarrow \mathbb{R}^{d}$ and $\sigma^{i}$ : $[0, T] \times \mathbf{W} \times U \rightarrow \mathbb{R}^{d \times d^{\prime}}$ satisfy (4.2) with the same $\alpha$ and $\int|x|^{2} \mu(d x)<+\infty$. Then the family $\left(X^{i}, K^{i}\right)_{i \in I}$ is tight in $C\left([0, T] ; \mathbb{R}^{d}\right) \times C\left([0, T] ; \mathbb{R}^{d}\right)$.

We would like to follow the same martingale formulation presented in the case of non-controlled MSDEs in order to find an optimal control, but we do not dispose of an appropriate metric on the space $L^{0}(0, T ; U)$ of measurable functions $u$ : $[0, T] \rightarrow U$ which could make possible the same compactness methods. Therefore, following an idea of [10], we imbed this space into a larger one and introduce relaxed controls.

Let $\lambda$ be the Lebesgue measure on $\mathcal{B}\left(\mathbb{R}_{+}\right)$. For $t \geq 0$ we consider

$$
\mathbf{U}_{t}:=\{q \text { measure on }[0, t] \times U \mid q(E \times U)=\lambda(E), \forall E \in \mathcal{B}([0, t])\}
$$

and denote $\mathbf{U}:=\mathbf{U}_{T}$. Recall that $\mathbf{U}_{t}$ is a compact metric space under the weak topology. This topology is the same (see [11, 3.2]) as the stable topology, i.e. the topology determined by the real-valued functions defined on $[0, t] \times U$ which are only bounded, measurable and continuous with respect to $v \in U$ (instead of continuous functions).

We will use the disintegration of an element $q \in \mathbf{U}$ given by $q(d s, d v)=$ $d s q^{\mathbf{d}}(s, d v)$, where $q^{\mathbf{d}}$ is a measurable kernel of mass 1 . We can embed the space $L^{0}(0, T ; U)$ in $\mathbf{U}$ by the formula

$$
q(d s, d v):=d s \delta_{u(s)}(d v), \quad u \in L^{0}(0, T ; U)
$$


$\left(\delta_{a}\right.$ is the Dirac measure concentrated in $a$ ). The space of these atomic measures is denoted $\mathbf{U}^{0}$.

If $t \in[0, T], q \in \mathbf{U}$, we let $\theta_{t}: \mathbf{U} \rightarrow \mathbf{U}_{t}, \theta_{t} q:=q_{\mid[0, t] \times U}$ and $\gamma_{t}:=\theta_{t}^{-1}\left(\mathcal{B}\left(\mathbf{U}_{t}\right)\right)$. Of course, $\left\{\gamma_{t}\right\}_{t \geq 0}$ is an increasing filtration on $\left(\mathbf{U}, \mathcal{B}(\mathbf{U})\right.$ ) (if $t>T$, let $\gamma_{t}:=$ $\mathcal{B}(\mathbf{U}))$.

Let $\left(\Omega, \mathcal{F}, P,\left\{\mathcal{F}_{t}\right\}_{t>0}\right)$ be a stochastic basis. We say that a random variable $Q: \Omega \rightarrow \mathbf{U}$ is adapted if $Q^{-1}\left(\gamma_{t}\right) \subseteq \mathcal{F}_{t}, \forall t \geq 0$. One can show that a random variable $Q$ is adapted if and only if it has a disintegration $Q^{\mathbf{d}}:[0, T] \times \Omega \rightarrow \mathcal{P}(U)^{\dagger}$ which is progressively measurable.

Additionally to (3.6), we introduce new notations:

$$
\begin{aligned}
\tilde{\Omega} & :=\bar{\Omega} \times \mathbf{U}, \quad \tilde{\mathcal{F}}:=\overline{\mathcal{F}} \otimes \mathcal{B}(\mathbf{U})=\mathcal{B}(\tilde{\Omega}) ; \\
\tilde{\mathcal{F}}_{t} & :=\mathcal{B}_{t} \otimes \mathcal{B}_{t} \otimes \gamma_{t}, \quad t \geq 0 ; \\
\tilde{\Omega}_{0} & :=\bar{\Omega}_{0} \times \mathbf{U} ; \quad \tilde{F}_{a}:=F_{a} \times \mathbf{U}, \quad a \geq 0 .
\end{aligned}
$$

If $R \in \mathcal{P}(\tilde{\Omega})$, we denote $\tilde{\mathcal{F}}^{R}:=\tilde{\mathcal{F}} \vee \mathcal{N}_{R}, \tilde{\mathcal{F}}_{t}^{R}:=\bigcap_{s>t}\left(\tilde{\mathcal{F}}_{s} \vee \mathcal{N}_{R}\right), t \geq 0$, where $\mathcal{N}_{R}$ is the family of $R$-negligible sets $\tilde{\mathcal{F}}$. The canonical processes $\tilde{X}, \tilde{K}, \tilde{Q}$ are defined by:

$$
\tilde{X}(x, \eta, q):=x, \quad \tilde{K}(x, \eta, q):=\bar{K}(x, \eta), \quad \tilde{Q}(x, \eta, q)=q,
$$

for $(x, \eta, q) \in \tilde{\Omega}$. We will use the following differential operator:

$$
\tilde{\mathcal{L}} f(t, x, v):=\frac{1}{2} \operatorname{tr} \sigma \sigma^{*}(t, x, v) D^{2} f(x(t))+\langle b(t, x, v), D f(x(t))\rangle,
$$

with $(t, x, v) \in[0, T] \times \mathbf{W} \times U$ and $f \in C^{2}\left(\mathbb{R}^{d}\right)$.

Definition. We say that a probability measure $R$ on $\tilde{\Omega}$ is a solution of the martingale problem for (4.1) if $R\left(\tilde{\Omega}_{0}\right)=1, R \circ \tilde{X}_{0}^{-1}=\mu$ and for each $f \in C_{c}^{2}\left(\mathbb{R}^{d}\right)$, the continuous process

$$
\begin{aligned}
\tilde{M}_{t}^{f}:= & f\left(\tilde{X}_{t}\right)-f\left(\tilde{X}_{0}\right)-\int_{[0, t] \times U} \tilde{\mathcal{L}} f(s, \tilde{X}, v) \tilde{Q}(d s, d v) \\
& +\int_{0}^{t}\left\langle D f\left(\tilde{X}_{s}\right), d \tilde{K}_{s}\right\rangle, \quad t \in[0, T],
\end{aligned}
$$

is a martingale under $\left(\tilde{\Omega}, \tilde{\mathcal{F}}^{R}, R,\left\{\tilde{\mathcal{F}}_{t}^{R}\right\}_{t \geq 0}\right)$.

In order to obtain a similar assertion to Proposition 3.5, we need the following result:

Lemma 4.2. There exists a progressively measurable process $\tilde{\sigma}:[0, T] \times \mathbf{W} \times$ $\mathcal{P}(U) \rightarrow \mathbb{R}^{d \times\left(d^{\prime}+d\right)}$ such that

$$
\tilde{\sigma} \tilde{\sigma}^{*}(t, x, \nu)=\int_{U} \sigma \sigma^{*}(t, x, v) \nu(d v), \quad \forall(t, x, \nu) \in[0, T] \times \mathbf{W} \times \mathcal{P}(U),
$$

${ }^{\dagger}$ For a topological space $S$, we denote $\mathcal{P}(S)$ the set of probability measures on $S$, on which the weak topology is considered. 
and

$$
\tilde{\sigma}\left(t, x, \delta_{v}\right)=\left[\sigma(t, x, v), \mathbf{0}_{d}\right] \in \mathbb{R}^{d \times d^{\prime}} \times \mathbb{R}^{d \times d}, \quad \forall(t, x, v) \in[0, T] \times \mathbf{W} \times U .
$$

Moreover, if (4.2) holds, then

$$
|\tilde{\sigma}(t, x, \nu)| \leq \alpha\left(1+\|x\|_{\mathbf{W}}\right), \quad \forall(t, x, \nu) \in[0, T] \times \mathbf{W} \times \mathcal{P}(U) .
$$

The approximative proof of this lemma can be found in [10, Th. 2.5].

We also define $\tilde{b}:[0, T] \times \mathbf{W} \times \mathcal{P}(U) \rightarrow \mathbb{R}^{d}$ by

$$
\tilde{b}(t, x, \nu):=\int_{U} b(t, x, v) \nu(d v), \quad(t, x, \nu) \in[0, T] \times \mathbf{W} \times \mathcal{P}(U) .
$$

Definition. We say that $\left(\Omega, \mathcal{F}, P,\left\{\mathcal{F}_{t}\right\}_{t \geq 0}, B, X, K, Q\right)$ is a relaxed control for (4.1) if $\left(\Omega, \mathcal{F}, P,\left\{\mathcal{F}_{t}\right\}_{t \geq 0}, B, X, K, Q^{\mathbf{d}}\right)$ is a control for the equation

$$
\left\{\begin{array}{l}
d X_{t}+A\left(X_{t}\right) d t \ni \tilde{b}\left(t, X, Q_{t}^{\mathbf{d}}\right) d t+\tilde{\sigma}\left(t, X, Q_{t}^{\mathbf{d}}\right) d B_{t}, \quad t \in[0, T] \\
X_{0} \stackrel{\text { law }}{=} \mu,
\end{array}\right.
$$

and $Q: \Omega \rightarrow \mathbf{U}$ is an adapted random variable with $Q^{\mathbf{d}}$ as a progressively measurable disintegration.

Proposition 4.3. Let $R$ be a solution of the martingale problem for (4.1). Then there exists an extension $\left(\Omega, \mathcal{F}, P,\left\{\mathcal{F}_{t}\right\}_{t \geq 0}\right)$ of $\left(\tilde{\Omega}, \tilde{\mathcal{F}}^{R}, R,\left\{\tilde{\mathcal{F}}_{t}^{R}\right\}_{t \geq 0}\right)$ (with projection $\theta)$ and a standard, $d^{\prime}+d$-dimensional Brownian motion $\bar{B}$ with respect to this extension such that $\left(\Omega, \mathcal{F}, P,\left\{\mathcal{F}_{t}\right\}_{t \geq 0}, B, X, K, Q\right)$ is a relaxed control, where $X:=\tilde{X} \circ \theta, K:=\tilde{K} \circ \theta$ and $Q:=\tilde{Q} \circ \theta$.

Conversely, if (4.2) holds, $\left(\Omega, \mathcal{F}, P,\left\{\mathcal{F}_{t}\right\}_{t \geq 0}, B, X, K, Q\right)$ is a relaxed control for (4.1), and $\int|x|^{2} \mu(d x)<+\infty$, then $P \circ(X, K, Q)^{-1}$ is a solution of the martingale problem.

The proof of this result follows in the same way as that of Proposition 3.5, after observing that

$$
\int_{[0, t] \times U} \tilde{\mathcal{L}} f(s, \tilde{X}, v) \tilde{Q}(d s, d v)=\int_{0}^{t} \overline{\mathcal{L}} f\left(s, \tilde{X}, Q_{s}^{\mathbf{d}}\right) d s,
$$

where

$$
\overline{\mathcal{L}} f(t, x, \nu):=\frac{1}{2} \operatorname{tr} \tilde{\sigma} \tilde{\sigma}^{*}(t, x, \nu) D^{2} f(x(t))+\langle\tilde{b}(t, x, \nu), D f(x(t))\rangle,
$$

for $(t, x, \nu) \in[0, T] \times \mathbf{W} \times \mathcal{P}(U)$ and $f \in C^{2}\left(\mathbb{R}^{d}\right)$.

Remark. If $\left(\Omega, \mathcal{F}, P,\left\{\mathcal{F}_{t}\right\}_{t>0}, \bar{B}, X, K, Q\right)$ is a relaxed control for equation (4.1) and $P \circ(X, K, Q)^{-1}\left(\bar{\Omega} \times \overline{\mathbf{U}}^{0}\right)=1$ then there exists a progressively measurable process $u$ such that $\left(\Omega, \mathcal{F}, P,\left\{\mathcal{F}_{t}\right\}_{t \geq 0}, B, X, K, u\right)$ is a control and $Q(d t, d v)=$ $d t \delta_{u_{t}}(d v), \forall t \in[0, T]$, a.s., where $B$ is the projection on $\mathbb{R}^{d}$ of $\bar{B}$. The converse also holds, enlarging eventually $\left(\Omega, \mathcal{F}, P,\left\{\mathcal{F}_{t}\right\}_{t \geq 0}\right)$ to an extension. 
Let $\tilde{\mathcal{R}}$ denote the family of probability measures on $\tilde{\Omega}$ which are solutions of the martingale problem for $(4.1)$ and $\tilde{J}: \mathcal{P}(\tilde{\Omega}) \rightarrow]-\infty,+\infty]$ defined by

$$
\tilde{J}(R):=\mathbb{E}_{R}\left[\int_{[0, T] \times U} g(t, \tilde{X}, v) \tilde{Q}(d t, d v)+h\left(\tilde{X}_{T}\right)\right] .
$$

Theorem 4.4. Suppose that (4.2), (4.3) hold, and $\int|x|^{2} \mu(d x)<+\infty$. If $b(t, \cdot, \cdot)$ and $\sigma(t, \cdot, \cdot)$ are continuous for every $t \in[0, T]$, then $\tilde{\mathcal{R}}$ is a compact set in $\mathcal{P}(\tilde{\Omega})$ and there exists $R^{*} \in \tilde{\mathcal{R}}$ such that $\tilde{J}\left(R^{*}\right)=\min \{\tilde{J}(R) \mid R \in \tilde{\mathcal{R}}\}$.

According to Proposition 4.3, this result asserts that there exists an optimal relaxed control for the problem of minimizing

$$
\mathbb{E}\left[\int_{0}^{T} g\left(t, X, Q_{t}^{\mathbf{d}}\right) d t+h\left(X_{T}\right)\right]
$$

over the class of relaxed controls $\left(\Omega, \mathcal{F}, P,\left\{\mathcal{F}_{t}\right\}_{t \geq 0}, B, X, K, Q\right)$ for equation (4.1).

Proof. By Theorem 4.1 and Proposition 4.3, $\left\{R \circ\left(\operatorname{pr}_{\tilde{\Omega}}^{\tilde{\Omega}}\right)^{-1} \mid R \in \tilde{\mathcal{R}}\right\}$ is tight, hence relatively compact in $\mathcal{P}(\bar{\Omega})$. The set $\mathcal{P}(\mathbf{U})$ is compact, and thus $\tilde{\mathcal{R}}$ is relatively compact in $\mathcal{P}(\tilde{\Omega})$. We have to prove that $\tilde{\mathcal{R}}$ is also closed. For that purpose, let us consider a sequence $\left(R_{n}\right)_{n \in \mathbb{N}^{*}} \subseteq \tilde{\mathcal{R}}$ which converges to $R_{0} \in \mathcal{P}(\tilde{\Omega})$.

As in the proof of Theorem 3.6, one can obtain $R_{0}\left(\tilde{\Omega}_{0}\right)=1, R_{0} \circ \tilde{X}_{0}^{-1}=$ $\mu$. It remains to show that for each $f \in C_{c}^{2}\left(\mathbb{R}^{d}\right), \tilde{M}^{f}$ is a martingale under $\left(\tilde{\Omega}, \tilde{\mathcal{F}}^{R}, R,\left\{\tilde{\mathcal{F}}_{t}^{R}\right\}_{t \geq 0}\right)$. But this is equivalent to

$$
\mathbb{E}_{R_{0}}\left(\tilde{M}_{t}^{f}-\tilde{M}_{s}^{f}\right) \Gamma=0, \quad \forall 0 \leq s \leq t \leq T,
$$

for every real-valued, bounded, continuous, $\tilde{\mathcal{F}}_{s}$-measurable function $\Gamma$.

Let us fix $f \in C_{c}^{2}\left(\mathbb{R}^{d}\right), \Gamma$ like above and $s, t \in[0, T], s \leq t$. It is not difficult to see that the function

$$
f\left(\tilde{X}_{t}\right)-f\left(\tilde{X}_{s}\right)-\int_{s}^{t} \tilde{\mathcal{L}} f(r, \tilde{X}, v) \tilde{Q}(d r, d v)
$$

is continuous and bounded on $\tilde{\Omega}$ (because $b(t, \cdot, \cdot)$ and $\sigma(t, \cdot, \cdot)$ are continuous, uniformly in $x \in \mathbf{W}$ with respect to $v \in U)$. Hence

$$
\mathbb{E}_{R_{n}}\left[\left(f\left(\tilde{X}_{t}\right)-f\left(\tilde{X}_{s}\right)-\int_{s}^{t} \tilde{\mathcal{L}} f(r, \tilde{X}, \tilde{Q}) d r\right) \Gamma\right]
$$

converges to

$$
\mathbb{E}_{R_{0}}\left[\left(f\left(\tilde{X}_{t}\right)-f\left(\tilde{X}_{s}\right)-\int_{s}^{t} \tilde{\mathcal{L}} f(r, \tilde{X}, \tilde{Q}) d r\right) \Gamma\right] .
$$

Finally, by Proposition 3.4,

$$
\lim _{n \rightarrow \infty} \mathbb{E}_{R_{n}}\left[\Gamma \int_{s}^{t}\left\langle D f\left(\tilde{X}_{r}\right), d \tilde{K}_{r}\right\rangle\right]=\mathbb{E}_{R_{0}}\left[\Gamma \int_{s}^{t}\left\langle D f\left(\tilde{X}_{r}\right), d \tilde{K}_{r}\right\rangle\right]
$$


(see also the proof of Theorem 3.6). In conclusion, $\mathbb{E}_{R_{0}}\left(\tilde{M}_{t}^{f}-\tilde{M}_{s}^{f}\right) \Gamma=0$ and from this relation it follows that $R_{0}$ solves the martingale problem.

To show that $\tilde{J}$ attains its minimum on $\tilde{\mathcal{R}}$ it is sufficient to prove that $\tilde{J}$ is lower semicontinuous. Let us consider $\bar{J}: \tilde{\Omega} \rightarrow]-\infty,+\infty]$, defined by

$$
\bar{J}(x, \eta, q):=\int_{[0, T] \times U} g(t, x, v) q(d t, d v)+h(x(T)), \quad(x, \eta, q) \in \tilde{\Omega} .
$$

If $\left(x_{n}, \eta_{n}, q_{n}\right) \rightarrow(x, \eta, q)$ in $\tilde{\Omega}$, then

$$
\liminf _{n \rightarrow \infty} \bar{J}\left(x_{n}, \eta_{n}, q_{n}\right) \geq \liminf _{n \rightarrow \infty} \int_{[0, T] \times U} g(t, x, v) q_{n}(d t, d v)+h(x(T)) \geq \bar{J}(x, \eta, q) .
$$

The first inequality holds by $(4.3)-i), i v)$ and the other by [11, Proposition 2.11] and $(4.3)-i i)$, $i i i)$. Hence, $\bar{J}$ is l.s.c., and, consequently, so is $\tilde{J}$.

Now we return to our initial optimal control problem; it turns out that under some convexity assumptions every relaxed control corresponds to a control with the same value of the cost functional.

We define

$$
\chi(t, x, v):=\left(b(t, x, v), \sigma \sigma^{*}(t, x, v), g(t, x, v)\right), \quad(t, x, v) \in[0, T] \times \mathbf{W} \times U .
$$

Theorem 4.5. Suppose that, in addition to the assumptions of Theorem 4.4, for all $(t, x) \in[0, T] \times \mathbf{W}, g(t, x, \cdot)$ is continuous and $\chi(t, x, U)$ is convex. Then the problem of minimizing (4.4) has an optimal control.

Proof. Let $R^{*}$ be an element of $\tilde{\mathcal{R}}$ which minimizes $\tilde{J}$, as in Theorem 4.4.

It is clear that, for every $\nu \in \mathcal{P}(U), \int_{U} \chi(t, x, v) \nu(d v) \in \chi(t, x, U)$, because $\chi(t, x, U)$ is compact and convex. Let us consider the $\sigma$-field of progressively measurable sets,

$$
\mathcal{P}:=\left\{E \subseteq[0, T] \times \tilde{\Omega} \mid E \cap([0, t] \times \tilde{\Omega}) \in \mathcal{B}[0, t] \otimes \tilde{\mathcal{F}}_{t}, \forall t \in[0, T]\right\} .
$$

The set

$$
\left\{(t, \omega, v) \in[0, T] \times \tilde{\Omega} \times U \mid \int_{U} \chi(t, \tilde{X}(\omega), v) Q_{t}^{\mathbf{d}}(\omega)(d v)=\chi(t, \tilde{X}(\omega), v)\right\},
$$

where $Q^{\mathrm{d}}$ is a progressively measurable disintegration of $\tilde{Q}$, belongs to $\mathcal{P} \otimes \mathcal{B}(U)$ and has nonempty sections for every $(t, \omega) \in[0, T] \times \tilde{\Omega}$. By using a measurable selection theorem (for example [7, Th. III.22]), one can show that there exists a process $u:[0, T] \times \tilde{\Omega} \rightarrow U$ which is $(\mathcal{P}, \mathcal{B}(U))$-measurable (that is, progressively measurable with respect to the filtration $\left.\left\{\tilde{\mathcal{F}}_{t}\right\}_{t \geq 0}\right)$ such that

$$
\int_{U} \chi(t, \tilde{X}(\omega), v) Q_{t}^{\mathbf{d}}(\omega)(d v)=\chi\left(t, \tilde{X}(\omega), u_{t}(\omega)\right), \quad d t R^{*}(d \omega) \quad \text { a.e. }
$$

(in fact, we obtain a measurable process with respect to the universal completion of $\mathcal{P}$, but this will coincide almost everywhere with a $\mathcal{P}$-measurable process). Denoting $Q(d t, d v):=d t \delta_{u_{t}}(d v)$, we have that $R:=R^{*} \circ(\tilde{X}, \tilde{K}, Q)^{-1}$ is an element 
of $\tilde{\mathcal{R}}$. By Proposition 4.3 and the remark below (since $R\left(\bar{\Omega} \times \mathbf{U}^{0}\right)=1$ ), it follows that there exists a control $\mathcal{U}=\left(\Omega, \mathcal{F}, P,\left\{\mathcal{F}_{t}\right\}_{t>0}, B, X, K, \bar{u}\right)$ for equation (4.1) such that $P \circ\left(X, K, Q_{\bar{u}}\right)^{-1}=R$, where $Q_{\bar{u}}(d t, d v):=d t \delta_{\bar{u}_{t}}(d v)$. Since

$$
J(\mathcal{U})=\tilde{J}(R)=\tilde{J}\left(R^{*}\right),
$$

it follows that $\mathcal{U}$ is an optimal control.

\section{References}

[1] I. Asiminoaiei, A. Răşcanu, Approximation and simulation of stochastic variational inequalities - splitting up method, Numer. Funct. Anal. Optim. 18 (3-4) (1997), 251282.

[2] V. Barbu, Optimal Control of Variational Inequalities, Research Notes in Mathematics 100, Pitman (Advanced Publishing Program), Boston, MA, 1984.

[3] A. Bensoussan, Lectures on stochastic control, in Nonlinear Filtering and Stochastic Control (Cortona, 1981). Lecture Notes in Math. 972, Springer, Berlin, (1982), 1-62.

[4] A. Bensoussan, A. Răşcanu, Parabolic variational inequalities with random inputs, in Les Grands Systèmes des Sciences et de la Technologie. RMA Res. Notes Appl. Math. 28, Masson, Paris, (1994), 77-94.

[5] A. Bensoussan, A. Răşcanu, Stochastic variational inequalities in infinitedimensional spaces, Numer. Funct. Anal. Optim. 18 (1-2) (1997), 19-54.

[6] H. Brézis, Opérateurs Maximaux Monotones et Semi-groupes de Contractions dans les Espaces de Hilbert, North Holland Mathematics Studies, No. 5. Notas de Matemática 50, North Holland Publishing Co., Amsterdam, 1973.

[7] C. Castaing, M. Valadier, Convex Analysis and Measurable Multifunctions, Lecture Notes in Math. 580, Springer Verlag, Berlin, 1977.

[8] E. Cépa, Équations différentielles stochastiques multivoques, in Séminaire de Probabilités, XXIX. Lecture Notes in Math. 1613, Springer, Berlin, (1995), 86-107.

[9] G. Ciucu, C. Tudor, Probabilităţi şi Procese Stocastice. Vol. I. Editura Academiei Republicii Socialiste România, Bucharest, 1978.

[10] N. El Karoui, D. Hủu Nguyen, M. Jeanblanc-Picqué, Compactification methods in the control of degenerate diffusions: existence of an optimal control. Stochastics 20 (3) (1987), 169-219.

[11] J. Jacod, J. Mémin, Sur un type de convergence intermédiaire entre la convergence en loi et la convergence en probabilité, in Seminar on Probability, XV (Univ. Strasbourg, Strasbourg, 1979/1980). Lecture Notes in Math. 850, Springer, Berlin, (1981), 529 546.

[12] I. Karatzas, S. E. Shreve, Brownian Motion and Stochastic Calculus, Graduate Texts in Mathematics, 113, Springer Verlag, New York, 1988.

[13] A. Răşcanu, Deterministic and stochastic differential equations in Hilbert spaces involving multivalued maximal monotone operators, Panamer. Math. J. 6 (3) (1996), 83-119.

[14] D. W. Stroock, S. R.S. Varadhan, Diffusion processes with continuous coefficients. IEII , Comm. Pure Appl. Math. 22 (1969), 345-400 and 479-530. 
[15] D. W. Stroock, S. R. S. Varadhan, Multidimensional Diffusion Processes, Grundlehren der Mathematischen Wissenschaften 233, Springer Verlag, Berlin, 1979.

[16] J. Yong, X. Y. Zhou, Stochastic controls. Hamiltonian Systems and HJB Equations, Applications of Mathematics, 43, Springer Verlag, New York, 1999.

[17] A. Zălinescu, Solutions faibles et contrôle optimal des inequations variationnelles stochastiques, Ph.D. thesis, Université de Brest, 2002.

[18] W. A. Zheng, Tightness results for laws of diffusion processes application to stochastic mechanics. Ann. Inst. H. Poincaré, Probab. Stat. 21 (2) (1985), 103-124.

Adrian Zălinescu

Institutul de Matematică "Octav Mayer"

Academia Română, filiala Iaşi

Bd. Carol I, nr. 8

RO-700505 Iaşi

Romania

e-mail: adrian.zalinescu@gmail.com

Received: 22 August 2007.

Accepted: 13 February 2008. 